\title{
Calculating melting temperature
}

Some journals still allow their contributors leisure, which makes for a readable account of a calculation of the Earth's inner core.

HAVE the journals in general, cven Nature perhaps, become so preoccupied with what is novel that their readers cannot hope to understand what is offered to them without first rushing to a textbook, a review or to some other device for catching up? The answer is almost certainly that they have. The question whether the convention is an impediment to the spread of knowledge is not answered as easily. People who need to find out are prepared to take the trouble; usually they succeed.

Nevertheless, some journals still fly in the face of what seems to have become the general conviction that all readers at all times know everything that has happened up to now, so that each of them can hit every first paragraph running, so to speak. One of these is the Geophysical Journal of the Royal Astronomical Society, which from time to time lets its contributors write chapters of textbooks as yet unwritten. One of the more interesting essays of this kind is an article by J.P. Poirier of the Institut de Physique de Globe in Paris (Geophys. J.R. astr. Soc. $85,315 ; 1986)$ which combines an account of a calculation of the temperature at the inner solid core of the Earth with a critical account of the dislocation theory of melting. Poirier's article is only 13 pages long.

The need for some discussion is clear. Even if the composition of the inner core and the surrounding molten outer core were known with certainty (which they are not), an accurate calculation of the relationship between the two would be beyond the scope of experimental investigation; the pressure and the temperature are too great. So it is necessary to bring all possible evidence to bear on some assumption of what the material of the inner and outer core consists of, to use the macroscopic properties of the Earth (mass, radius, moment of inertia and so on) to estimate the density as a function of depth and then to fix conditions at the boundary between the inner and outer cores by attempting to calculate the melting curve of the material there, the relationship between its melting temperature and the pressure. But conditions near the centre of the Earth are so far away from experimental ken that the faceless equations of thermodynamics, which assume that evidently variable quantities are constant, simply do not apply. At some stage, only a model of the phenomenon of melting will help.
But what model? It is curious how the Lindemann theory of melting, essentially an extension of Debye's theory of lattice vibrations in the early 1920 s, is usually the first to come to mind. (Lindemann, Churchill's chief scientific adviser during the Second World War who was afterwards made Lord Cherwell, would probably prefer to have been remembered for having learned how to correct stalling in First World War aircraft.) But this is the crudest device, based on the assumption that solids will melt when atomic vibrations become so robust that atoms are forever colliding with each other. Its thermodymanic error is that it takes no account of the free energy of the molten state.

The essence of melting is that it must be a cooperative phenomenon, one in which the whole or at least a substantial part of a melting solid is involved. For that is what the thermodynamics imply: at melting, there are two phases in equilibrium (one a solid and the other a liquid) whose energy density may be substantially different, as different as the latent heat, and which must be compensated for by the difference of entropy or of disorder between the two phases. Lindemann's theory might have been made into a more persuasive argument if, for example, he had sought to calculate how one collision between a pair of atoms in the vibrating lattice of a solid will increase the chance that one of the pair will promptly engage in another collision.

Poirier's tack is a dislocation theory of melting (there is an infinity of variations) based on the view that solids accumulate a greater density of linear dislocations in their lattice structure as they approach their melting point. That the occurrence of dislocations within an otherwise regular solid is a dynamic process is, of course, well attested; give a collection of atoms enough energy and one of them will surely lodge in the wrong place, distorting the patterns of its neighbours. The classical dislocations of the $1930 \mathrm{~s}$, involving the displacement of successive atoms in a line from the "true" positions, are energetically more easily formed. But the greater the density of dislocations, the less the sense of order within a lattice and the more like a liquid it appears on a microscopic scale. The extreme view is that a liquid is merely a solid saturated with dislocations.

The interest of Poirier's mini-textbook on the subject of dislocation melting is that he puts previous attempts at a comprehensive theory of melting in a sceptical perspective. Most people seem to have recognized that, apart from the energy embodied in an isolated dislocation, there is also an energy of interaction between them, but only some models have taken account of the entropy arising from different arrangements of configurations. The physical strain within a solid occasioned by the presence of dislocation works both ways, first by increasing the volume of a solid on the way to melting but also by reducing the strain energy of later displacements. And some people, it seems, seem to have been content to leave out of the account the change of volume there must be when a solid melts.

The point of all this is to choose the most suitable dislocation theory of melting for a calculation of the equilibrium between the inner and the outer core of the Earth; Poirier settles for a theory due to Ninomiya, published in 1978 . For what it is worth, Lindemann's approximation stands up well, but the theory is also nicely verified by its predictions for the properties of melting iron, certainly the predominant material in the inner solid core, under laboratory conditions.

What of the core itself? The best estimate seems to be that the temperature at the inner core boundary, that between solid nearly pure iron (with nickel as the principal impurity) and the outer molten core (which may have lighter elements as impurities) is within $100 \mathrm{~K}$ or so of 6,150 $\mathrm{K}$. This value is in the range covered by previous estimates, and for the time being there is probably little to choose between them except that Poirier provides such a detailed account of how, by his calculations, the melting temperature should change with pressure so that it should be possible to wring some relevant information from the seismic measurements.

The picture of the inner Earth that emerges from the calculations is not very different from that now widely accepted. Both at the boundary between the mantle and the core (at a depth of just under 3,000 $\mathrm{km}$ ) and at the boundary between the inner and the outer cores (at a depth of just over $5,000 \mathrm{~km}$ ) there are temperature discontinuities. The former, a drop of several hundred degrees $K$ between the liquid outer core and the base of the mantle, is easily enough accounted for by latent heat. That there should be a jump as large between the outer and the inner cores is more surprising, but consistent.

John Maddox 